\title{
Forcing linearity numbers for coatomic modules
}

\author{
Peter R. Fuchs ${ }^{1 *}$
}

\begin{abstract} commutative ring with identity if and only if $n \in\{0,1,2, \infty\} \cup\{q+2 \mid q$ is a prime power $\}$.

Keywords: Homogeneous functions, Forcing linearity numbers, Coatomic modules

2010 AMS: Primary 13C05, Secondary 16D10

${ }^{1}$ Inst. for Algebra, Johannes Kepler University, Austria

*Corresponding author: peter.fuchs@jku.at

Received: 19 July 2018, Accepted: 19 September 2018, Available online: 30 September 2018
\end{abstract}

We show that an integer $n \in \mathbb{N} \cup\{0\}$ is the forcing linearity number of a coatomic module over an arbitrary

\section{Introduction}

Throughout this paper $\mathrm{R}$ shall denote a commutative ring with identity and $\mathrm{V}$ a unital right $\mathrm{R}$-module. Consider the set $M_{R}(V):=\{f: V \rightarrow V \mid f(v r)=f(v) r$ for all $r \in R, v \in V\}$. Under the operations of pointwise addition and composition of functions, $M_{R}(V)$ is a near-ring with identity, called the near-ring of homogeneous functions. Note that $M_{R}(V)$ contains the endomorphism ring $\operatorname{End}_{R}(V)$. The question arises how much linearity is needed on a function $f \in M_{R}(V)$ to ensure that $f$ is linear on all of $V$, i.e. $f \in \operatorname{End}_{R}(V)$. More precisely, we say that a collection $\left\{W_{i} \mid i \in I\right\}$ of proper submodules forces linearity on $V$, if whenever $f \in M_{R}(V)$ and $f$ is linear on each $W_{i}, i \in I$, then $f \in \operatorname{End}_{R}(V)$. Thus $M_{R}(V)=\operatorname{End}_{R}(V)$ if and only if the empty collection forces linearity on $V$. The smallest number of modules which force linearity on $V$ gives rise to the forcing linearity number of $V$.

Definition 1.1. [3] Let $V$ be an $R$-module. The forcing linearity number $f \ln (V) \in \mathbb{N} \cup\{0, \infty\}$ of $V$ is defined as follows:

1. If $M_{R}(V)=\operatorname{End}_{R}(V)$, then $f \ln (V)=0$.

2. If $M_{R}(V) \neq \operatorname{End}_{R}(V)$, and there is some finite collection $\left\{W_{i} \mid 1 \leq i \leq n\right\}, n \in \mathbb{N}$, of proper submodules of $V$ which forces linearity on $V$, but no collection of fewer than $n$ proper submodules forces linearity, then we say that $f \ln (V)=n$.

3. If neither 1. or 2. holds, then we say that $f \ln (V)=\infty$.

Forcing linearity numbers have been found for several classes of rings and modules, see for example [3], [4], [5] and their references. In section 2 we determine the forcing linearity number of coatomic modules over an arbitrary commutative ring $R$ with identity. An $R$-module $V$ is called coatomic, if every proper submodule is contained in a maximal submodule of $V$. For example a finitely generated module or a semisimple module over any ring is coatomic. For a commutative noetherian local ring, the coatomic modules have been characterized in [7].

\section{Forcing linearity numbers of coatomic modules}

For an $R$-module $V$ and subsets $S_{1}, S_{2}$ of $V$ let $\left(S_{1}: S_{2}\right)=\left\{r \in R \mid S_{2} r \subseteq S_{1}\right\}$. For $v \in V$ let Ann $(v)=\{r \in R \mid v r=0\}$. 
Theorem 2.1. Let $V$ be an $R-$ module and let $M, N$ be maximal submodules of $V, M \neq N$. The following are equivalent:

1. The collection $\{M, N\}$ does not force linearity.

2. $\exists w \neq 0 \in V:(M: V)=(N: V)=\operatorname{Ann}(w)$.

Proof. $1 \Rightarrow 2$ : Since $\{M, N\}$ does not force linearity on $V$, there exists a function $f \in M_{R}(V)$ such that $f$ is linear on the submodules $M, N$, but $f \notin \operatorname{End}_{R}(V)$. Let $u, v \in V$ be such that $w:=f(u+v)-f(u)-f(v) \neq 0$. Since $M \neq N$, and $M, N$ are maximal, we have that $M+N=V$. For every $v \in V-M,(M: v)=(M: V)$, therefore $(M: V)$ and $(N: V)$ are maximal ideals. If $(M: V) \neq(N: V)$, then $(M: V)+(N: V)=R$, hence $r+s=1$ for some $r \in(M: V), s \in(N: V)$. Now $w r=f(u r+v r)-f(u r)-f(v r)=f(u r)+f(v r)-f(u r)-f(v r)=0$, since $f$ is linear on $M$. Similarly, ws $=0$, hence $w=w .1=w(r+s)=0$, a contradiction. Thus $(M: V)=(N: V)$, and since $(M: V) \subseteq A n n(w)$ and $(M: V)$ is a maximal ideal, it follows that $(M: V)=\operatorname{Ann}(w)$.

$2 \Rightarrow 1:$ Let $v \in V-M$. Then $(M: v)=(M: V)=A n n(w)$ and $h: V / M \rightarrow R w, h(v r / M):=w r$ is an isomorphism. Define a function $f: V \rightarrow V$ as follows: For $m \in M, n \in N$ let

$$
f(m+n):= \begin{cases}h(n / M) & \text { if } m+n \notin M \cup N \\ 0 & \text { otherwise }\end{cases}
$$

Since $M+N=V, f$ is defined on $V$. We show that $f$ is well-defined. Suppose $m_{1}+n_{1}=m_{2}+n_{2}, m_{1}, m_{2} \in M, n_{1}, n_{2} \in N$. If $m_{1}+n_{1} \in M \cup N$, then $f\left(m_{1}+n_{1}\right)=f\left(m_{2}+n_{2}\right)=0$. If $m_{1}+n_{1} \notin M \cup N$, then $n_{1} / M=n_{2} / M$, hence $f\left(m_{1}+n_{1}\right)=h\left(n_{1} / M\right)=$ $h\left(n_{2} / M\right)=f\left(m_{2}+n_{2}\right)$. Next we show that $f$ is homogeneous. Let $S:=V-(M \cup N)$. If $m+n \in S$, then $(N: m)=(N: V)$ and $(M: n)=(M: V)$. By our assumption $(M: V)=(N: V)=\operatorname{Ann}(w) \neq R$, hence $(N: m)=(M: n)$. If $r \notin(M: n)$, then $r \notin(N: m)$, which implies that $(m+n) r=m r+n r \in S$, hence $f((m+n) r)=h(n r / M)=h(n / M) r=f(m+n) r$. If $r \in(M: n)$, then $(m+n) r \notin S$, hence $f(m+n) r=h(n / M) r=h(n r / M)=h(0)=0=f((m+n) r)$. Now suppose $m+n \notin S$. Then $m+n \in M \cup N$, hence $(m+n) r \in M \cup N$ for all $r \in R$. Thus $f(m+n) r=0=f((m+n) r)$. It now follows that $f \in M_{R}(V)$. Since $f|M=f| N=0, f$ is linear on $M$ and $N$. However, for $m \in M-N$ and $n \in N-M$, we have that $m+n \in S$, thus $f(m+n)=h(n / M) \neq 0$, since $h$ is an isomorphism, whereas $f(m)+f(n)=0$, so $f \notin E n d_{R}(V)$. Therefore the collection $\{M, N\}$ does not force linearity on $V$.

For an $R$-module $V$ let $\operatorname{Rad}(V)$ denote the Jacobson radical of $V$ and let $J:=\operatorname{Rad}(R)$. Recall that an $R-$ module $V$ is called local, if $V$ contains a unique maximal submodule.

Theorem 2.2. For a noncyclic coatomic module $V$, the following are equivalent:

1. $f \ln (V)>2$.

2. $I:=(\operatorname{Rad}(V): V)$ is a maximal ideal and $I=A n n(w)$ for some

$0 \neq w \in V$.

Proof. $1 \Rightarrow 2:$ Let $\mathbf{M}$ denote the collection of all maximal submodules of $V$. Since $V$ is coatomic, $\mathbf{M} \neq \emptyset$. If there exist $M_{1}, M_{2} \in$ M such that $\left(M_{1}: V\right) \neq\left(M_{2}: V\right)$, then by Theorem 2.1 the collection $\left\{M_{1}, M_{2}\right\}$ forces linearity on $V$. Thus $\left(M_{1}: V\right)=\left(M_{2}: V\right)$ for all $M_{1}, M_{2} \in \mathbf{M}$ and $I=\bigcap\{(M: V) \mid M \in \mathbf{M}\}=(M: V)$ for all $M \in \mathbf{M}$, hence $I=(\operatorname{Rad}(V): V)$ is a maximal ideal. Like in the proof of Theorem 1 , we see that $I=\operatorname{Ann}(w)$ for some $w \neq 0$.

$2 \Rightarrow 1$ : Suppose that $V$ is a local module with unique maximal submodule $M$. Let $v \in V-M$. If $v R \neq V$, then $v R$ is contained in a maximal submodule, which implies $v R \subseteq M$, a contradiction. Consequently $v R=V$ for all $v \in V-M$, which contradicts our assumption that $V$ is noncyclic. Therefore there exist at least two maximal submodules. Suppose $f \ln (V) \leq 2$. Then there exists a collection of submodules $\left\{S_{1}, S_{2}\right\}$ which forces linearity on $V$. Since $V$ is coatomic, there exist maximal submodules $M_{1}, M_{2}$ such that $S_{1} \subseteq M_{1}, S_{2} \subseteq M_{2}$. Without loss of generality we may assume that $M_{1} \neq M_{2}$ (otherwise we can choose another maximal submodule, since $V$ is not local). Then $\left\{M_{1}, M_{2}\right\}$ also forces linearity on $V$. We have $(\operatorname{Rad}(V): V) \subseteq\left(M_{1}: V\right) \neq R$. By our assumptions $(\operatorname{Rad}(V): V)$ is a maximal ideal, hence $(\operatorname{Rad}(V): V)=\left(M_{1}: V\right)=\left(M_{2}: V\right)$. Also, $(\operatorname{Rad}(V): V)=A n n(w)$ for some $0 \neq w \in V$. Therefore $\left\{M_{1}, M_{2}\right\}$ does not force linearity by Theorem 1 , a contradiction.

Theorem 2.3. Let $V$ be coatomic. Suppose $I:=(\operatorname{Rad}(V): V)$ is a maximal ideal of $R$ and there exists $0 \neq w \in V$ such that $I=\operatorname{Ann}(w)$. Then

$$
f \ln _{R}(V)=f \ln _{R / I}(V / \operatorname{Rad}(V))
$$


Proof. We first show that $f \ln _{R / I}(V / \operatorname{Rad}(V)) \leq f \ln _{R}(V)$. Let $\left\{W_{i} \mid i \in I\right\}$ be a collection of proper submodules which forces linearity on $V$. Since $V$ is coatomic, we may assume that each $W_{i}, i \in I$, is maximal. We show that the collection $\left\{W_{i} / \operatorname{Rad}(V) \mid i \in\right.$ $I\}$ forces linearity on $V / \operatorname{Rad}(V)$. Suppose that this is not the case. Then there exists a homogeneous function $f: V / \operatorname{Rad}(V) \rightarrow$ $V / \operatorname{Rad}(V)$, which is linear on each submodule $W_{i} / \operatorname{Rad}(V), i \in I$, but not linear on $V / \operatorname{Rad}(V) . \operatorname{Let} \pi_{M}: V / \operatorname{Rad}(V) \rightarrow V / M$ denote the projection of $V / \operatorname{Rad}(V)$ onto $V / M$ for a maximal submodule $M$. Since $f$ is not linear, there exists a maximal submodule $M$ of $V$ such that $\pi_{M} f: V / \operatorname{Rad}(V) \rightarrow V / M$ is not linear. Since $I$ is a maximal ideal, $I=(M: V)$, hence $w(M: V)=0$, which implies $V / M \simeq w R$. Thus we obtain a homogeneous map $f_{1}: V / \operatorname{Rad}(V) \rightarrow w R$, which is linear on each submodule $W_{i} / \operatorname{Rad}(V), i \in I$. If $g: V \rightarrow V$ is defined by $g(v):=f_{1}(v / \operatorname{Rad}(V))$, then $g \in M_{R}(V)$ and linear on each $W_{i}, i \in I$, but not linear on $V$, a contradiction to our assumption that $\left\{W_{i} \mid i \in I\right\}$ forces linearity on $V$. For the reverse inequality suppose first that $f \ln _{R / I}(V / \operatorname{Rad}(V)) \leq 1$. Since $V / \operatorname{Rad}(V)$ is a vector space over the field $R / I$, it follows from Theorem 3.1 in [3] that $\operatorname{dim}_{R / I}(V / \operatorname{Rad}(V))=1$. Note that $\operatorname{Rad}(V)$ is a superfluous submodule, since $V$ is coatomic. It follows that $V$ is cyclic, hence $f \ln _{R / I}(V / \operatorname{Rad}(V))=0=f \ln (V)$. If $\operatorname{dim}_{R / I}(V / \operatorname{Rad}(V))=2$ or $f \ln _{R / I}(V / \operatorname{Rad}(V)) \geq 2$ and $R / I$ is infinite, we have that $f \ln _{R / I}(V / \operatorname{Rad}(V))=\infty$ by Theorem 3.1 in [3]. So suppose that $f \ln _{R / I}(V / \operatorname{Rad}(V)) \geq 3$ and $|R / I|=: q \in \mathbb{N}$. By [3], 3.8 and 3.10, $f \ln _{R / I}(V / \operatorname{Rad}(V))=q+2$. Choose $\left\{r_{1}, \ldots, r_{q}\right\} \subseteq R$ such that $R / I=\left\{r_{1} / I, \ldots, r_{q} / I\right\}$. It suffices to give a collection of $q+2$ proper submodules which forces linearity on $V$. Let $\left\{b_{i} \mid i \in I\right\} \subseteq V$ be such that $\left\{b_{i} / \operatorname{Rad}(V) \mid i \in I\right\}$ is a basis of the vector space $V / \operatorname{Rad}(V)$. As we have seen above, $|I| \geq 3$, so we can choose pairwise different elements $i_{1}, i_{2}, i_{3} \in I$. Let $\langle X\rangle$ denote the submodule generated by a subset $X \subseteq V$, and define $S_{1}:=\left\langle b_{i_{1}}, b_{i_{2}}\right\rangle+\operatorname{Rad}(V), S_{2}:=\left\langle b_{i_{1}}+b_{i_{3}}\right\rangle+\left\langle b_{i} \mid i \notin\left\{i_{1}, i_{3}\right\}\right\rangle+\operatorname{Rad}(V)$, and for $r \in\left\{r_{1}, \ldots, r_{q}\right\}$ define $S_{r}:=\left\langle b_{i_{1}}+r b_{i_{2}}, b_{i_{1}}+b_{i_{3}}\right\rangle+\left\langle b_{i} \mid i \notin\left\{i_{1}, i_{2}, i_{3}\right\}\right\rangle+\operatorname{Rad}(V)$. Note that all submodules are proper, since $\operatorname{Rad}(V)$ is superfluous. Similarly as in Theorems 3.8,3.10 in [3], one can prove that the collection $\left\{S_{1}, S_{2}\right\} \cup\left\{S_{r_{i}} \mid\right.$ $i \in\{1, \ldots, q\}\}$ forces linearity on $V$.

For $R$ local and $J$ T-nilpotent, Theorem 2.3 has been proved in [4], Theorem 5.1. The following example shows that Theorem 2.3 is not true in general, if $I$ is not the annihilator of some $0 \neq w \in V$.

Example 2.4. Let $R:=F[[x]]$ denote the ring of formal power series over a field $F$ and let $V:=R \times R$. Since $R$ is local with radical $J=(x), \operatorname{Rad}(V)=V J=(x) \times(x)$ and $I=(\operatorname{Rad}(V): V)=(x)$ is maximal. By [3], Corollary 2.4, $f \ln _{R}(V)=1$. However, $f \ln _{R / I}(V / \operatorname{Rad}(V))=f \ln _{F}\left(F^{2}\right)=\infty$, by [3], Theorem 3.1.

Theorem 2.5. Let $n \in \mathbb{N} \cup\{0, \infty\}$. Then $n$ is the forcing linearity number of a coatomic module over a commutative ring if and only if $n \in\{0,1,2, \infty\} \cup\{q+2 \mid q$ is a prime power $\}$.

Proof. It is well-known that there exist coatomic modules $V$ over a commutative ring $R$ such that $f \ln _{R}(V) \in\{0,1,2, \infty\}$, see for example [5]. If $V$ is a cyclic module, then $M_{R}(V)=E n d_{R}(V)$, hence $f \ln _{R}(V)=0$. Now suppose $f \ln _{R}(V)>2$. By Theorem 2.2, $I=(\operatorname{Rad}(V): V)$ is a maximal ideal and $I=A n n(w)$ for some $0 \neq w \in V$. By Theorem $2.3, f \ln _{R}(V)=f \ln _{R / I}(V / \operatorname{Rad}(V))$ and as we have remarked previously, $f \ln _{R / I}(V / \operatorname{Rad}(V)) \in\{\infty\} \cup\{q+2 \mid \mathrm{q}$ is a prime power $\}$.

It is not known to the author, whether Theorem 2.5 is true for every module over a commutative ring.

There is a class of rings which have the property that every right module is coatomic, or which is easily seen to be equivalent, every nonzero right module has a maximal submodule.

Definition 2.6. A ring $R$ is called a right max-ring, if every right $R$-module is coatomic. See [6].

Theorem 2.7. [2] For a commutative ring $R$, the following are equivalent:

1. $R$ is a max-ring.

2. $J$ is $T$-nilpotent and $R / J$ is von Neumann regular.

Theorem 2.8. Let $V$ be a module over a commutative max-ring $R$. If $R$ is not local, then $f \ln _{R}(V) \leq 2$.

Proof. Suppose that $R$ is not local, but $f \ln (V)>2$. Since $R$ is a max-ring, it follows from Theorem 2.7 and from [1], Proposition 18.3 that $\operatorname{Rad}(V)=V J$. By Theorem 2.2, $(\operatorname{Rad}(V): V)=(V J: V)$ is a maximal ideal. We have $J \subseteq(V J: V)$. Suppose that there exists an element $r \in(V J: V)-J$. Then $r \notin M$ for some maximal ideal $M$ of $R$. Let $R_{M}, V_{M}$ denote the localisations of $R, V$ at $M$. By [1], Proposition 18.3, $\operatorname{Rad}\left(V_{M}\right)=V_{M} J_{M}$. Since $R$ is a max-ring $J$ is T-nilpotent, thus $J_{M}$ is T-nilpotent. It follows from Theorem 2.5 that $R_{M}$ is a max-ring, hence $\operatorname{Rad}\left(V_{M}\right)=V_{M} J_{M} \neq V_{M}$. So let $w / 1 \in V_{M}-\operatorname{Rad}\left(V_{M}\right)$. From $r \in(V J: V)$, $w / 1 \cdot r / 1=w r / 1 \in V_{M} J_{M}$. Since $r \notin M, r / 1$ is invertible in $R_{M}$, hence $w / 1 \in V_{M} J_{M}=\operatorname{Rad}\left(V_{M}\right)$, a contradiction. It now follows that $J=(V J: V)$ is a maximal ideal of $R$, which contradicts our assumption that $R$ is not local. 


\section{References}

[1] C. Faith, Algebra. II. Ring theory. Grundlehren der Mathematischen Wissenschaften, No. 191. Springer-Verlag, BerlinNew York, 1976.

[2] R.M.Hamsher, Commutative rings over which every module has a maximal submodule, Proc. Amer. Math. Soc. 18 (1967), $1133-1137$.

[3] C.J.Maxson, J.H.Meyer, Forcing linearity numbers, J.Algebra 223 (2000), 190-207.

[4] C.J.Maxson, A.B.Van der Merwe, Forcing linearity numbers for modules over rings with nontrivial idempotents, J.Algebra 256 (2002), 66-84.

[5] C.J.Maxson, A.B.Van der Merwe, Forcing linearity numbers for finitely generated modules, Rocky Mountain J.Math.35 (3) (2005), 929-939.

[6] A.A. Tuganbaev, Rings whose nonzero modules have maximal submodules, J.Math.Sci. (New York) 109 (2002), no.3, $1589-1640$

[7] H.Zöschinger, Koatomare Moduln, Math.Z. 170 (1980), 221-232 